Research Article

\title{
Experimental and Numerical Analysis of Torque Properties of Rotary Elastomer Particle Damper considering the Effect of Gap and No Gap between Rotor and Body of the Damper
}

\author{
Allah Rakhio $\mathbb{D D}^{1}{ }^{1}$ Yasushi Ido, ${ }^{1}$ Yuhiro Iwamoto, ${ }^{1}$ and Atsushi Toyouchi ${ }^{2}$ \\ ${ }^{1}$ Department of Electrical and Mechanical Engineering, Nagoya Institute of Technology, Nagoya 466-8555, Japan \\ ${ }^{2}$ KYB Cooporation, 25478 Dota, Kani-shi, Gifu 509-0298, Japan \\ Correspondence should be addressed to Allah Rakhio; r.allah.778@stn.nitech.ac.jp
}

Received 26 July 2021; Revised 22 September 2021; Accepted 29 September 2021; Published 18 October 2021

Academic Editor: Xuping Zhang

Copyright (c) 2021 Allah Rakhio et al. This is an open access article distributed under the Creative Commons Attribution License, which permits unrestricted use, distribution, and reproduction in any medium, provided the original work is properly cited.

Particle dampers are devices used to control the vibration of mechanical systems. In this research, prototypes of rotary elastomer particle dampers are experimentally tested considering gap and no gap between shaft and cylinder. There is a gap between the rotor and cylinder in the gap model; particles can move from one chamber to another. There is no space for the particles to move from one chamber to another in the no-gap model. Elastomer particles are soft, and they have different behavior from hard (metallic) particles. Experiments on rotary elastomer particle dampers considering the gap between rotor and cylinder helped investigate the effects of the change in packing fraction, rotational speed, size of elastomers, and the gap between the rotor and the damper body. A numerical simulation approach based on the discrete element analysis method is used to perform a quantitative and qualitative analysis of the rotary elastomer particle damper. The simulation results are in great agreement with the experiment results. It is observed that packing fraction, rotational speed, size of elastomer particles, and the gap between rotor and cylinder play a vital role in producing higher damper torque.

\section{Introduction}

Vibrations are frequent oscillations of mechanical systems. Most of the case vibrations are unwanted excitations to the mechanical systems. Vibrations can be extremely dangerous for mechanical systems which can severely affect health as well as human lives. In addition, vibrations decrease the efficiency and accuracy of mechanical devices and machines. Researchers have been trying to develop better techniques and methods to suppress unwanted vibrations. The passive vibration damping technique is one of the most suitable solutions in industrial applications of vibration control. Conventional passive damping technique consists of frictional devices, impact dampers, oil dampers, and tuned mass dampers. In conventional dampers, viscoelastic materials are used. Viscoelastic materials degrade and disintegrate with time and environmental temperature. Similarly, the main problem with oil dampers is the leakages. A particle damper is a vibration control device in which different metals, ceramic, tungsten, rubbers, sand, or other types of particles are used. Particle damper controls the vibration of any mechanical system by dissipating the kinetic energy; kinetic energy can be dissipated in two ways in a particle damper, one by friction and second is collisions between particleparticle and particle-wall of the damper.

There are two types of dampers used in the particle-based vibration control system. One is particle impact damper, and the other is the piston and rotor-based particle damper. Particle impact dampers consist of a closed cavity with particles inside it. In piston and rotor-based particle dampers, a moving rod or rotor is used inside the container of the damper. The piston-based particle damper is a linear damper consists of a rod, and a rotary particle damper consists of a rotating shaft.

In recent decades, researchers and scientists turned their focus towards particle dampers due to several advantages of 
particle dampers, such as no loss in efficiency at higher temperature $[1,2]$ and simple construction [3], which results in ease of maintenance and manufacturing [4].

Previous work has mainly focused on using metallic or other hard particles in the particle damper [5]. Marhadi and Kinra proposed a particle damper for beam [6], the effect of packing fraction was investigated. In their experiments, the particles made of lead, steel, tungsten, and glass were used. They argued that the size and the number of particles are the parameters that must be considered while designing particle impact dampers. Sánchez et al. studied the effect of different sizes and shapes of grains; they observed the welding of grains at high temperatures [7]. Liu et al. proposed a method of vibration control of structures with the help of particle dampers; they considered the nonlinear characteristics of the damper [8]. Their investigation proved that the damping is affected by the thickness of the disk cavity. Researchers also used the tungsten powder as a damping medium in the damper [9]. It was shown that packing fraction and container dimension have an enormous influence on the damping capabilities of a damper. Friend and Kinra reported the nonlinear behavior of particle dampers using lead particles [10]. Also, they showed the high damping capability of lead compared to other metallic materials. Fabian et al. showed particle impact damper's applications in automobile applications [11]. They investigated the oil pan noise reduction in a combustion engine using a granular particle damper. Kachare and Bimleshkumar [12] researched on the effect of packing fraction and size of metallic particles; they used $1 \mathrm{~mm}, 2 \mathrm{~mm}$, and $3 \mathrm{~mm}$ diameter spherical shaped particles made of copper. They concluded that $1 \mathrm{~mm}$ diameter-sized particle showed better performance than $2 \mathrm{~mm}$ and $3 \mathrm{~mm}$ diameter particles, and at $50 \%$ packing fraction, the damper shows maximum performance; after 50\% packing fraction, a decline in damper performance was noticed.

In recent years, elastomer particles have been introduced and studied [1]. Metal particles and elastomer particles are different in nature and properties, so they behave differently in different situations. More importantly, one of the essential advantages of using elastomer particles is noise reduction [13]. Very few articles have been published on the damping behavior of elastomer particles; most of them are considering linear elastomer particle dampers. Morishita et al. proposed a double rod type linear particle damper. They used elastomer particles instead of metallic ones [14]. They reported that the change of packing fraction, frequency, and stroke of the piston changes the damper force. Kawamoto et al. proposed a double rod type linear damper using elastomer particles made of silicon [1]. Kishan et al. investigated torque dependency on the angle of the rotor in a rotary elastomer particle damper [15].

As for our knowledge, no more articles have been found on the damper torque properties of a rotary elastomer particle damper. It is essential to note that hard metallic particles or powders were used in most previous studies. Although the metallic particles are primarily used in the dampers as a vibration suppression medium, the main issue with metallic particles is the high cost and noise due to collisions. In this research, we are using elastomer particles instead of metallic ones. Elastomer particles do not produce noise because of their soft and elastic properties. Several factors can affect the damping performance of particle dampers, such as packing fraction and size of particles. However, due to the change in these parameters, there was a massive change observed in damping properties of damper because these changes can cause a change in the cooperative moment of damping particles [16]. The gap between the rotor and cylinder can also significantly change the cooperative moment of particles inside the damper. The performance of a particle damper is affected by the packing fraction and size of particles [17]. None of the studies examined the role of the gap between the rotor and the cylinder in a rotary elastomer particle damper. So, it is essential to do an in-depth study on the behavior of elastomer particles considering the effect of the gap between the rotor and damper body, which can affect the damping capabilities of a rotary elastomer particle damper. This research provides new insight into the research on particle dampers. In addition, the findings of this research can help academia and industry to design more efficient rotors for particle damper applications. This paper proposes rotary elastomer particle dampers with gap and no gap between rotor and cylinder.

It is expected that the rotary elastomer particle damper with the gap between rotor and cylinder can provide a strong damper torque than the rotary elastomer particle damper with no gap between rotor and cylinder. Rotary elastomer particle damper with no-gap model produces torque due to resistance of particles due to friction and collision between particle-particle and particle-wall of the damper, but in case of rotary elastomer particle damper with gap model, extra layers of strongly compressed elastomer particles can enter the gap between the rotor and the body. Because of the elastic properties of the elastomer particles, the particles in the gap region can provide high repulsive forces in the motion of the rotor. There is a direct correlation between repulsive forces from the particles and damper torque.

In this study, the damper torque characteristics of rotary elastomer particle damper were investigated. The effects of packing fraction, rotor speed, size of elastomer particles, and the gap between rotor and cylinder on damper torque were examined. The behavior of elastomer particles inside the damper was analyzed by numerical simulations using the discrete element method.

\section{Experiments}

2.1. Fabrication of Elastomer Particles. TSE3466 (produced by Momentive Performance Materials) is the main ingredient for preparing silicon rubber particle samples. TSE3466 has a hardness of 60 , the density is $1.10 \mathrm{~kg} / \mathrm{m}^{3}$, and the tensile strength $(\mathrm{MPa})$ is 7.4. TSE3466-A is the primary raw material, but it is mixed with TSE3466-B, with a ratio of $10: 1$. Product B worked as a catalyst, and its job is to speed up the drying procedure. Before the molding, the air from the raw material was removed by a vacuum chamber. In the next step, we removed the dried elastomers from the molds after 24 hours. We prepared elastomer particles of $3 \mathrm{~mm}, 4 \mathrm{~mm}$, 
and $5 \mathrm{~mm}$ diameter for experimental purposes. The main reason to select particles of $3 \mathrm{~mm}, 4 \mathrm{~mm}$, and $5 \mathrm{~mm}$ diameter is the expected higher damper torque of the damper due to higher deformation range comparing to particles of $2 \mathrm{~mm}$ and $1 \mathrm{~mm}$ diameter. Qualitative analysis of $3 \mathrm{~mm}, 4 \mathrm{~mm}$, and $5 \mathrm{~mm}$ diameter particles is more accessible than smaller particles. Fabrication of $1 \mathrm{~mm}$ and $2 \mathrm{~mm}$ diameter particles is more complex than the $3 \mathrm{~mm}$ diameter particles; it takes a long time to remove smaller size particles from the molds. Figure 1 shows the prepared samples after being removed from the molds. Figure 1 shows the sample of a spherical particle of $3 \mathrm{~mm}, 4 \mathrm{~mm}$, and $5 \mathrm{~mm}$ diameter particles made from TSE3466.

2.2. Damper Construction and Working. The schematic diagram of the damper used in this experiment is shown in Figure 2; the damper consisted of the body, cover, rotor, holder, shaft, and bearings. All dimensions in the figure are in millimeters $(\mathrm{mm})$. Two different types of rotors were used, as shown in Figure 2. In this damper, the holder's job was to hold the damper while performing experiments; it stopped the damper body from rotation. There were two tapered roller bearings (NTN30202) used in the damper, which supported the rotor of the damper. The parts other than the bearings were made of stainless steel SAE304. The damper was tested with no particles inside, and it produced no damping torque, so it can be stated that bearings and damper parts do not affect the test results. In the study, the effect of packing fraction on the rotary elastomer particle damper is analyzed. The packing fraction = total mass of particles/ (density of particles and volume of the damper container). The working mechanism of the damper is simple. As the force applied to the rotor of the damper, the rotor started to move. As the rotor moved, it pushed the elastomer particles to move in the direction of the rotor. The main reason behind selecting this damper is its simple working mechanism, which helped to understand and answer the article's primary research question. This damper size is suitable for several mechanical engineering applications, i.e., car seat suspension or automobile door closer. In the future, the prototype can easily be tested for practical application due to its small size.

2.3. Experiential Setup and Experimental Conditions. The damper torque of the designed elastomer particle damper is measured experimentally using an experimental setup shown in Figure 3. This test setup consists of a servo motor (Model-NXM920A-PS10) from Oriental Motors Co., Ltd., Japan. The experimental setup has rotational speed ranging from 0 to $120 \mathrm{rpm}$ of the shaft. Couplings are used to connect the damper shaft with the shaft of the test setup. The torque meter used in the experiment is of UTM II from Unipulse Corporation with a torque monitoring capacity of $10 \mathrm{Nm}$. The torque meter measured the resistive torque generated by the damper. A control unit is used to control the rotation of the motor. The interface is used to provide the torque readings from the torque meter and transfer them to the computer. The maximum standard deviation was $0.13(\mathrm{Nm})$.

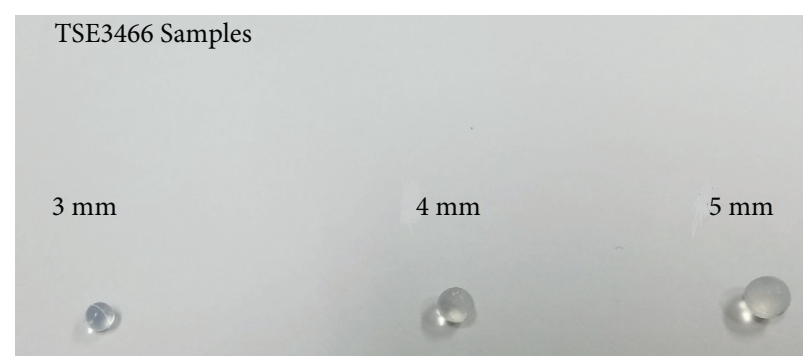

FIgURE 1: Elastomer samples of TSE3466 $3 \mathrm{~mm}, 4 \mathrm{~mm}$, and $5 \mathrm{~mm}$ diameter particles after being removed from molds.

Initially, 5 to 6 revolutions at high rpm were given to the shaft to settle down the particles at random locations. The temperature of the cylinder of the damper was monitored during the experiments; few degrees rise in temperature was detected; however, it did not change the mechanical properties of TSE3466, so the effect of temperature can be neglected.

\section{Numerical Simulation of the Rotary Elastomer Particle Damper}

The discrete element method (DEM) is used to simulate the particle behavior inside the rotary elastomer particle damper. In DEM, the collision between the particle-particle and particle-wall is modeled based on contact force laws. The DEM simulation provides a much satisfying insight into the particle governing process. The DEM simulations can enhance basic understanding of particle behavior, as well as it can help to improve the design and efficiency of particle dampers. A theoretical approach was used to prepare this model by considering two particles which we named particle $i$ and particle $j$.

The basic equations of motions can be stated as

$$
\begin{gathered}
m_{i} \frac{\mathrm{d}^{2} r_{i}}{\mathrm{~d} t^{2}}=F_{i}, \\
I_{i} \frac{\mathrm{d} \Omega_{i}}{\mathrm{~d} t}=T_{i},
\end{gathered}
$$

where $t$ is the time, $m_{i}$ is the mass of the particle, $r_{i}$ is the position vector of the particle $i, F_{i}$ is the sum of the forces acting on the particle $i, I_{i}$ is the moment of inertia, $\Omega_{i}$ is the angular velocity vector, and $T_{i}$ represents the total torque applied on the particles. In these equations, a particle is named particle $i$, by considering which the model will further progress. $F_{i}, I_{i}, T_{i}$ can be expressed as

$$
\begin{aligned}
& F_{i}=F+F_{c t}+F_{g}, \\
& T_{i}=r_{i} \times F_{c t}, \\
& I_{i} \frac{8}{15} \rho \pi a^{5}, \\
& F_{g}=m_{i} g
\end{aligned}
$$




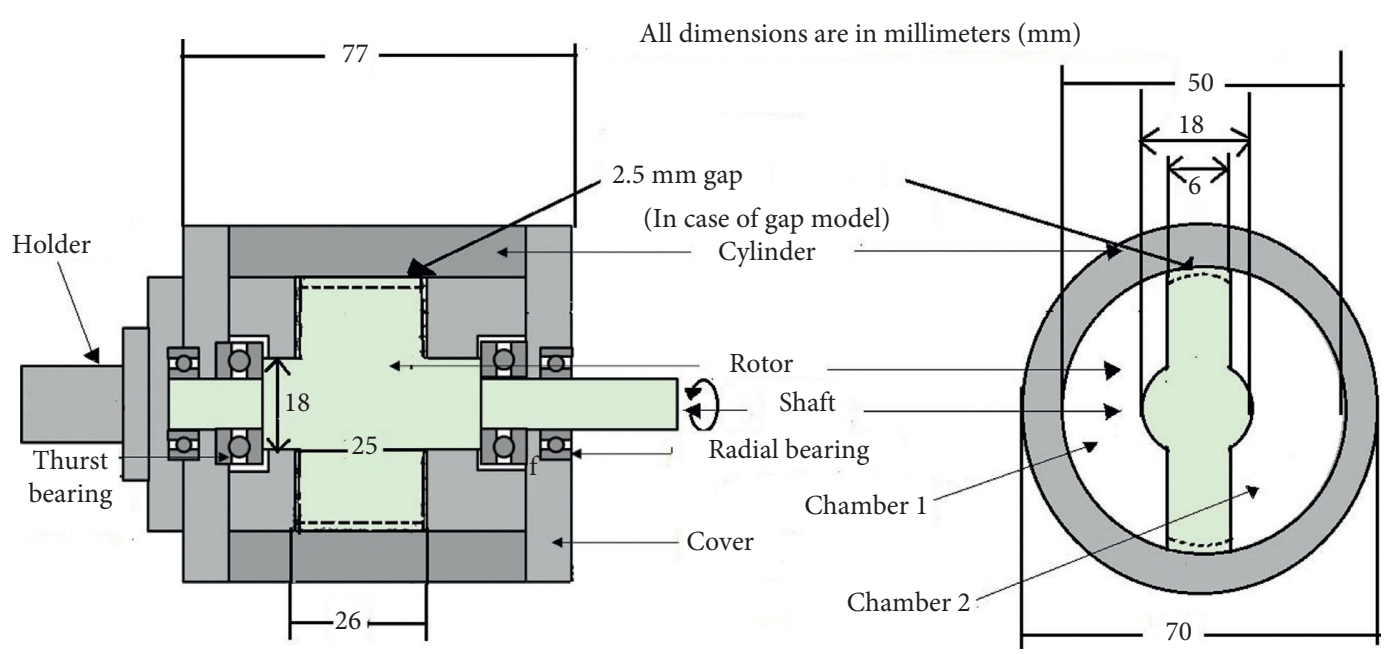

(a)

(b)

Figure 2: Schematic diagram of the rotary damper with gap and no gap between the rotor and cylinder; all dimensions are in millimeters $(\mathrm{mm})$. (a) Cross-sectional side views of the damper. (b) Cross-sectional top views of the damper.

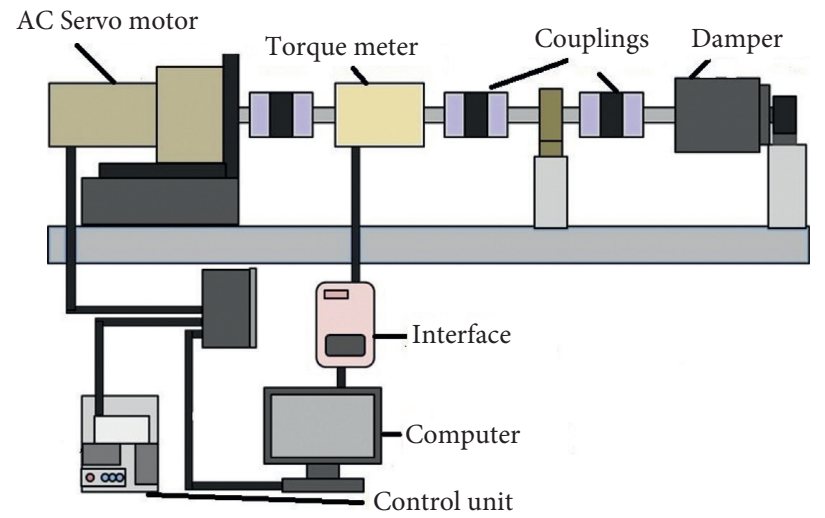

FIgURE 3: Schematic diagram of experimental setup.

where $F_{c n}$ is the normal contact force between particles or the walls of the damper, $F_{c t}$ is the force in the tangential direction, $F_{g}$ is the gravity force, $g$ is the gravitational acceleration vector, $a$ is the radius of the elastomer particle, and $\rho$ is the particle density. This model is based on the Kelvin-Voigt material model, which consists of an elastic spring dashpot and slider.

$$
\begin{aligned}
& F_{c n}=\left(-K_{n} \delta_{n}+C_{n} V_{i j} \cdot n_{i}\right) n_{i}, \\
& F_{c t}=K_{t} \delta_{t}+C_{t} V_{f i j},
\end{aligned}
$$

where the subscript $n$ represents the parameters of normal direction, whereas the subscript $t$ represents the parameters of tangential direction, $K_{n}$ is the elastic modulus in the normal direction, $K_{t}$ is the elastic modulus in the tangential direction, $C_{n}$ is the viscosity coefficient in the normal direction, $C_{t}$ is the viscosity coefficient in the tangential direction, $\delta_{n}$ is the strain in the normal direction, $\delta_{t}$ is the strain in the tangential direction, $n_{i}$ is the unit vector in the normal direction from $i$ to particle $j, V_{i j}$ is the relative velocity vector of particle $i$ with respect to particle $j$, and $V_{f i j}$ is the tangential relative velocity.

$$
\begin{aligned}
V_{i j} & =v_{i}=v_{j}, \\
V_{f i j} & =V_{i j}-\left(V_{i j} \cdot n_{i}\right) n_{i}+2 a\left(\omega_{i}-\omega j\right) \times n_{i},
\end{aligned}
$$

where $\omega_{i}$ and $\omega_{j}$ are the angular velocity vectors of particles $i$ and $j$, respectively. Viscosity coefficients in the normal direction $C_{n}$ and viscosity coefficient in the tangential direction $C_{t}$ can be represented as [18]

$$
\begin{aligned}
& C_{n}=\bar{\alpha} \sqrt{m_{i} K_{n} \delta_{n}^{0.25}}, \\
& C_{t}=\bar{\alpha} \sqrt{m_{i} K_{n} \delta_{c t}^{0.25}},
\end{aligned}
$$

where $\delta_{c t}$ is the displacement in elastomer particle in the tangential direction; $\bar{\alpha}$ determines the magnitude of viscous damper force, which can be expressed by the following equation [18]: 


$$
\bar{\alpha}=2.2 \sqrt{\frac{\ln \left(e^{2}\right)}{\ln \left(e^{2}\right)+\pi^{2}}} .
$$

The elastic modulus in the normal direction is expressed by the following equation based on Hertz's contact theory [18]:

$$
\begin{aligned}
K_{n i j} & =\frac{2}{3 \pi}\left(\frac{1}{\delta_{i}}\right) \sqrt{\frac{a \delta_{n}}{2}}, \\
K_{n i w} & =\frac{4}{3 \pi}\left(\frac{1}{\delta_{i}+\delta_{w}}\right) \sqrt{a \delta_{n}}, \\
\delta_{i} & =\frac{1-v_{i}^{2}}{E_{i} \pi}, \\
\delta_{w} & =\frac{1-v_{w}^{2}}{E_{w} \pi},
\end{aligned}
$$

where the subscript $w$ indicates that the quantities relate to the wall, $K_{n i j}$ is the elastic coefficient when particle $i$ and particle $j$ are in contact, whereas $K_{\text {niw }}$ is the elastic coefficient when the particle $i$ is in contact with the wall of the damper, $E_{i}$ and $E_{w}$ are the moduli of longitudinal elasticity of the particle and wall, respectively, and $v_{i}$ and $v_{w}$ are the Poisson's ratios of the particle and wall, respectively. Considering that there is no slip at the point of contact, the tangential elastic coefficient can be expressed as the following equation [18]:

$$
\begin{aligned}
K_{t i j} & =\left(\frac{2 \sqrt{2 a} G_{i}}{2-v_{i}}\right) \delta_{n}^{0.5}, \\
K_{t i w} & =\left(\frac{8 \sqrt{a} G_{i}}{2-v_{i}}\right) \delta_{n}^{0.5},
\end{aligned}
$$

where $K_{t i j}$ is the elastic coefficient when particle $i$ is in contact with another particle $j, K_{t i w}$ is the elastic coefficient when particle $i$ is in contact with the wall of the damper, and $G_{i}$ is the transverse elastic modulus of the particle and is expressed as follows [18]:

$$
G_{i}=\frac{E_{i}}{2\left(1+v_{i}\right)} .
$$

For calculating the time steps of the velocity, displacement, and angular velocity of the particles, the Adams-Bashforth method of second-order accuracy is applied. The analytical model for the rotary elastomer particle damper is the same as the rotary damper prepared for experiments, as shown in Figure 2. The simulations are conducted at the packing fractions of $30 \%, 40 \%, 45 \%$, and $50 \%$. The rotational speed of the shaft in the simulation is kept at $60 \mathrm{rpm}$. The material of the elastomer particles is silicon rubber (TSE3466). The diameter of the elastomer particles used in the numerical simulations is $3 \mathrm{~mm}$. The friction coefficient is 0.5 in this simulation [19], and the time step is 0.0000000075 seconds. The density of the particle is $1.10 \mathrm{~kg} / \mathrm{m}^{3}$. The particle's Poisson ratio and wall's Poisson ratio were 0.5 and 0.3 .

One of the limitations of this simulation is long processing time. Data collection of simulation at each packing fraction took around 4 to 5 days. More powerful computers can reduce the computational time of particle-based simulation systems. Advanced and fast parallel processing can make more efficient use of the algorithm.

\section{Results and Discussion}

4.1. Torque Generation Mechanism of the Rotary Elastomer Particle Damper. We have performed extensive experiments to analyze the damper torque performance of rotary elastomer particle dampers. The qualitative and quantitative analysis of the rotary damper is also performed via numerical simulation. The purpose of the simulation is to allow us to analyze the behavior of the elastomer particles inside the damper. We first present a simulation experiment result to study the behavior of particles, followed by a detailed analysis of the damper torque properties using rotary elastomer particle damper with gap and no-gap model prototypes. Figures 4 and 5 show the torque versus time graph of the gap and no-gap models of the rotary elastomer particle damper. Figures 4 and 5 show the simulation results and the experimental results of rotary elastomer particle damper at $60 \mathrm{rpm}$ and $50 \%$ packing fraction using particles of $3 \mathrm{~mm}$ diameter. To investigate the effect of particle deformation and particle sliding, decomposition of both the factors based on simulation results is conducted. The decomposition results are taken from $50 \%$ packing fraction at $60 \mathrm{rpm}$, using $3 \mathrm{~mm}$ diameter elastomer particles. In Figure 6 , the torque due to particle deformation is higher than the torque due to sliding friction. The dominant source of torque generation is particle deformation, so the compression force on particles plays a significant role in producing higher damper torque. Particles under higher compressive forces tend to generate strong repulsive forces in the rotation of the rotor. Due to repulsive forces from the particles, damper torque is produced by the damper.

Figure 7 shows the experimental and simulation results of the torque versus packing fraction curve using elastomer particles of $3 \mathrm{~mm}$ diameter in the no-gap model. The results are taken from the rotational speed of $60 \mathrm{rpm}$. In Figure 7, the simulation results are in good agreement with experimental results. The experimental curve shows slightly higher torque than simulation results; this may be because of the surface roughness factor of the particles, which is not considered in the simulation. In our future research, this difference between experimental and simulation results can be reduced by considering the effect of the surface roughness factor in simulations.

The design with gap and no gap between rotor and cylinder produced no noise during experiments. Due to the elastic nature of elastomer particles, they deform on collision and compression but do not produce noise. Due to the collision between elastomer particles, the sound is inaudible 


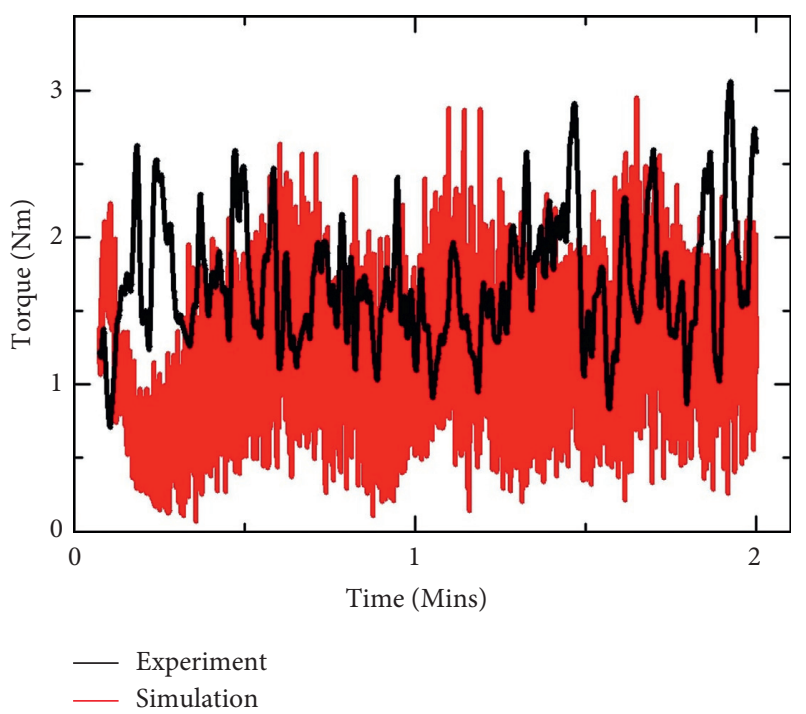

FIGURE 4: Time history of the rotary damper with a gap model using $3 \mathrm{~mm}$ diameter particles; the packing fraction is $50 \%$, and the rotational speed is $60 \mathrm{rpm}$.

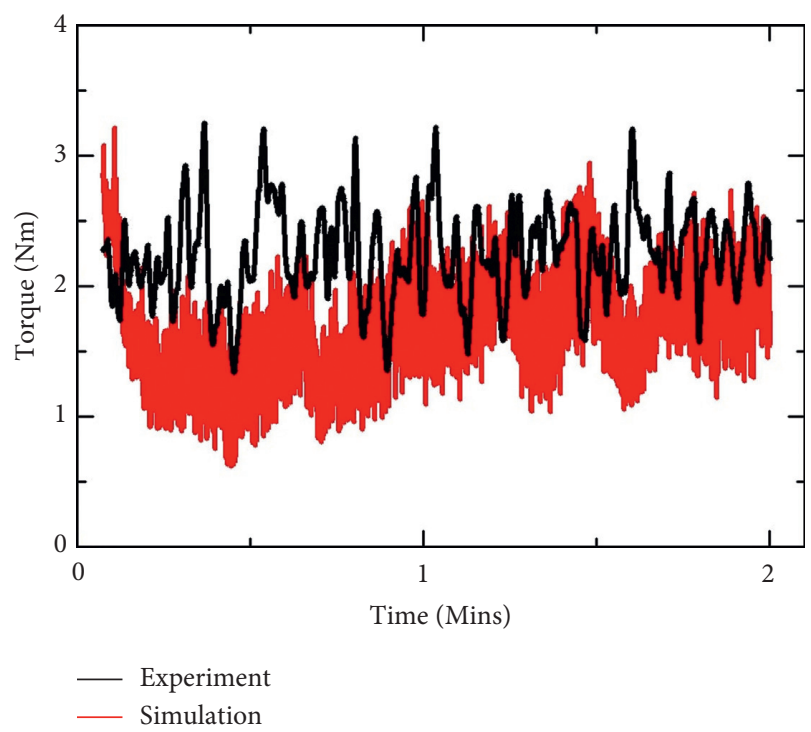

FIGURE 5: Time history of the rotary damper with a no-gap model using $3 \mathrm{~mm}$ diameter particles; the packing fraction is $50 \%$, and the rotational speed is $60 \mathrm{rpm}$.

to humans, but it may be possible to record or measure it using a microphone.

Figures 8 and 9 show the qualitative results obtained from the simulation of rotary elastomer particle damper with no-gap and gap models. To investigate the force of compression on the elastomer particles, the simulation was conducted at $50 \%$ packing fraction at the rotational speed of $60 \mathrm{rpm}$, using $3 \mathrm{~mm}$ diameter elastomer particles.

In Figures 8 and 9, the strength of compressive force on the particles depends on the rotor's location inside the cylinder. When the rotor rotates, it forces the elastomer particles to move forward in the direction of the rotor

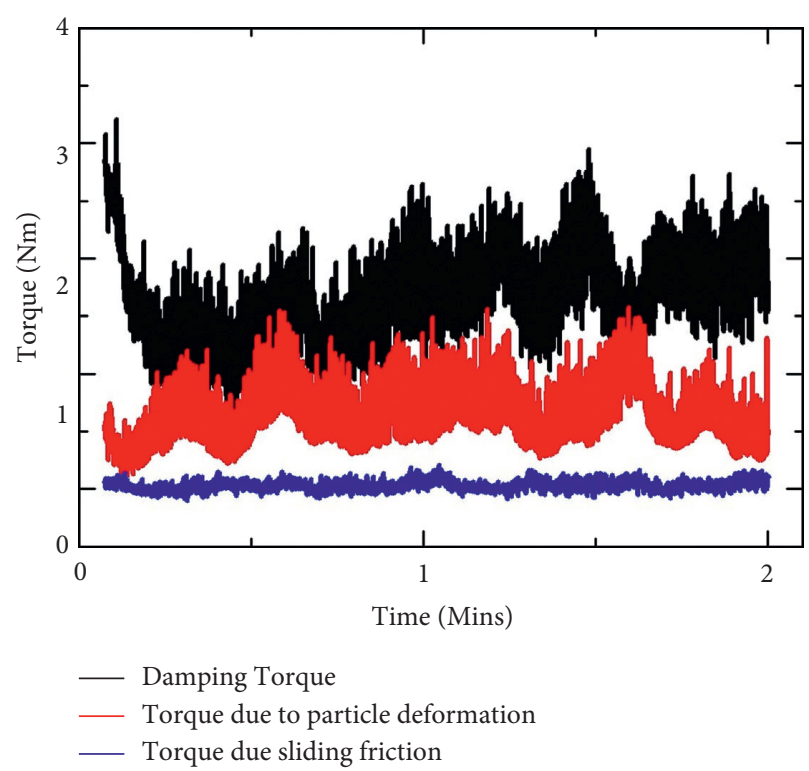

FIgURE 6: Time history of the rotary damper with a no-gap model using $3 \mathrm{~mm}$ diameter particles, decomposition of torque due to particle deformation, and torque due to sliding friction.

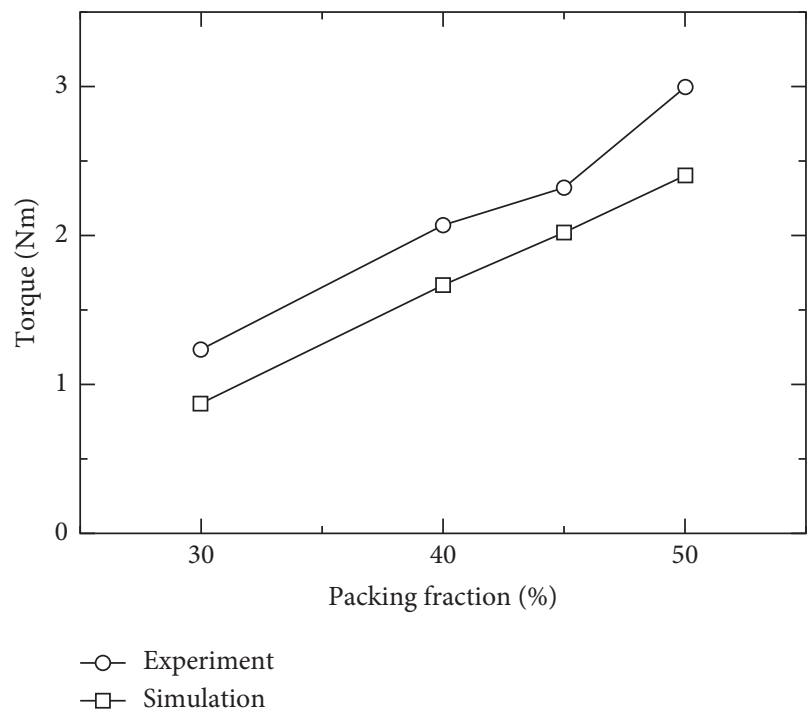

FIgURE 7: Experimental and simulation results of the torque versus packing fraction curve using elastomer particles of $3 \mathrm{~mm}$ diameter in the no-gap model. The rotational speed is $60 \mathrm{rpm}$.

rotation. Particles in front of the rotor push other particles to move forward. The force of compression on the elastomer particles is investigated using simulation results. In Figures 8 and 9, several particles have a strong force of compression near the rotor, due to which a strong repulsive force from the elastomer particles is produced. When the rotor is at the bottom area of the cylinder, the force of compression is strong because the gravitational force is also acting on the particles. The elastic repulsive force of particles is produced by the collision of elastomer particles and frictional forces between particle-particle and particle-wall. It is important to 

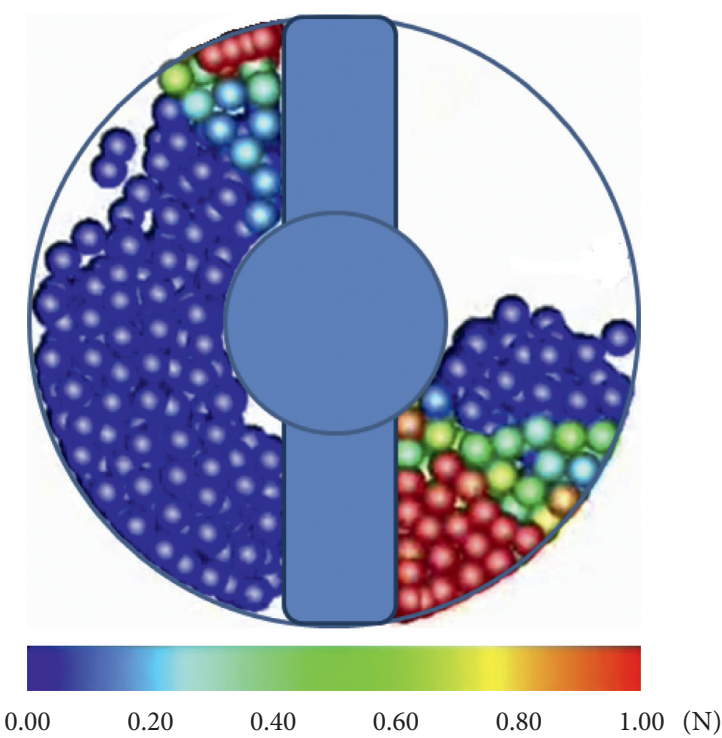

(a)

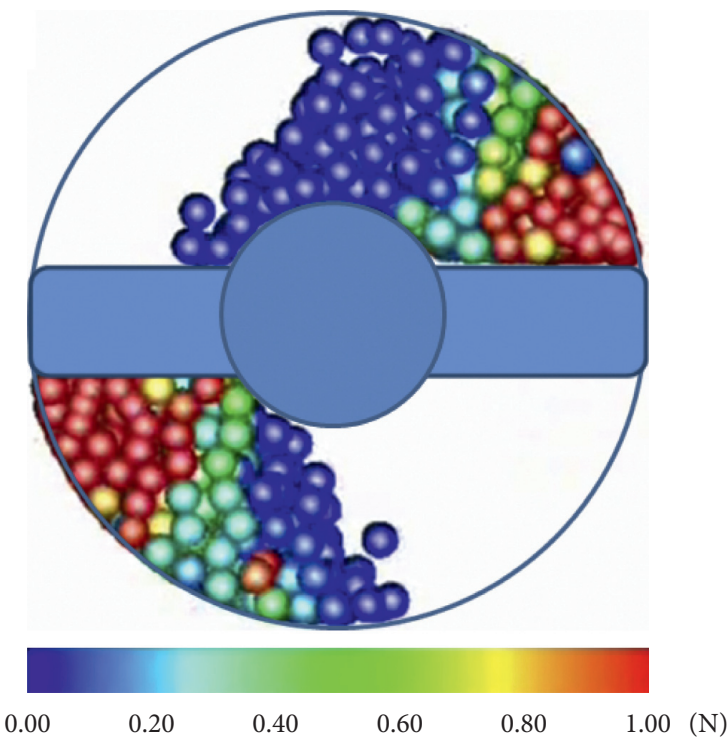

(c)

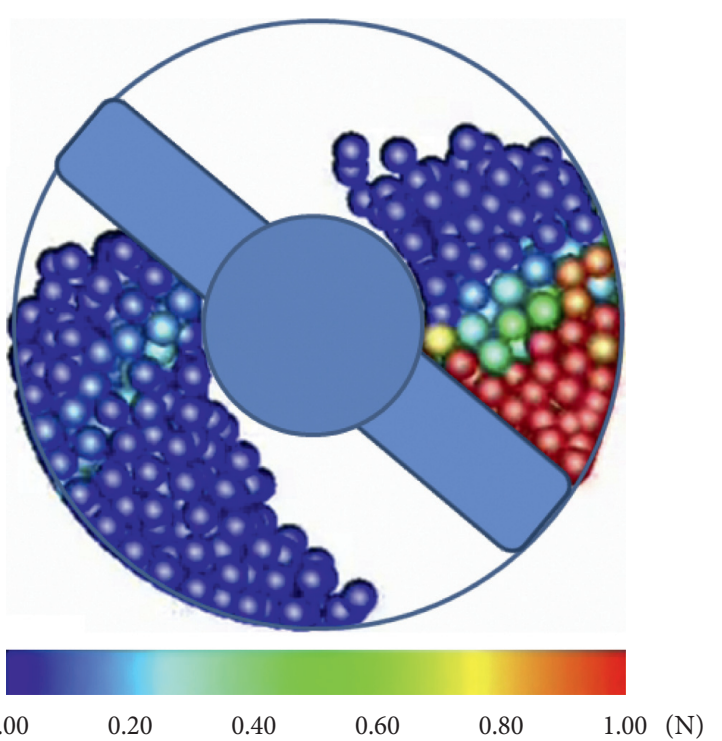

(b)

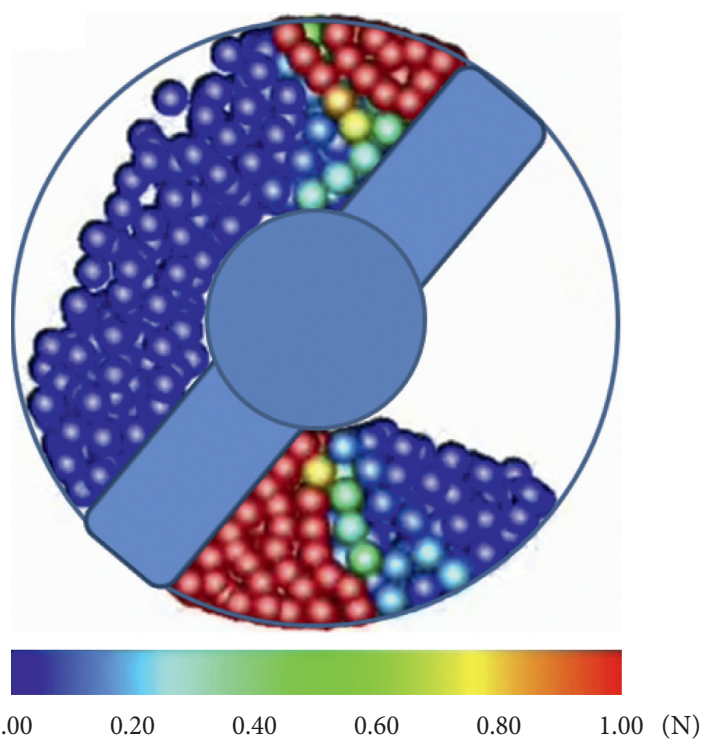

(d)

FIGURE 8: Compressive force acting on the particle inside the rotary damper with the no-gap model; the packing fraction is $50 \%$, and the rotational speed is $60 \mathrm{rpm}$. (a) 0 degree, (b) 45 degrees, (c) 90 degrees, and (d) 135 degrees. The direction of rotation is counter-clockwise.

mention that the rotary damper with the gap between rotor and cylinder and no gap between rotor and cylinder may look similar, but in the case of the gap model, due to the gap between the damper body and the rotor, an extra layer of elastomer particles is formed. In the gap between rotor and cylinder, particles are under a strongly compressed state, resulting in the generation of a strong friction zone inside the damper.

4.2. Effect of Packing Fraction and Rotor Speed on Damper Torque of the Damper. Packing fraction can be defined as the mass of the particles divided by the volume of the damper and the density of the particles [1]. Rotary elastomer particle damper shows an increase in damper torque with the increase of packing fraction. Figure 10 shows the torque vs. packing fraction graph of no-gap models of rotary elastomer particle dampers. Figure 11 shows the torque vs. packing fraction graph of gap models of rotary elastomer particle dampers. Similar observations are made in gap and no-gap models of rotary elastomer particle dampers; they both showed an increase in damper torque with the increase of packing fraction. The main reason is that the number of particles decreases inside the damper with a decrease in packing fraction [20]. A small variation in particle number leads to a large change in damping behavior. A decrease in the number of particles increases the volume of void gaps in the rotary elastomer particle damper. When elastomer particles are moved forward by the rotor at a high packing fraction, the particles do not have void spaces available. 

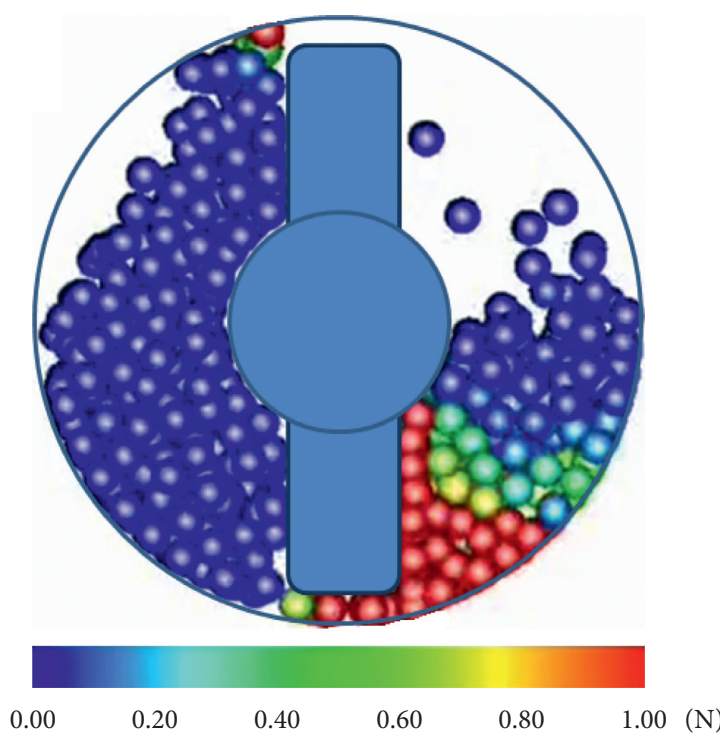

(a)

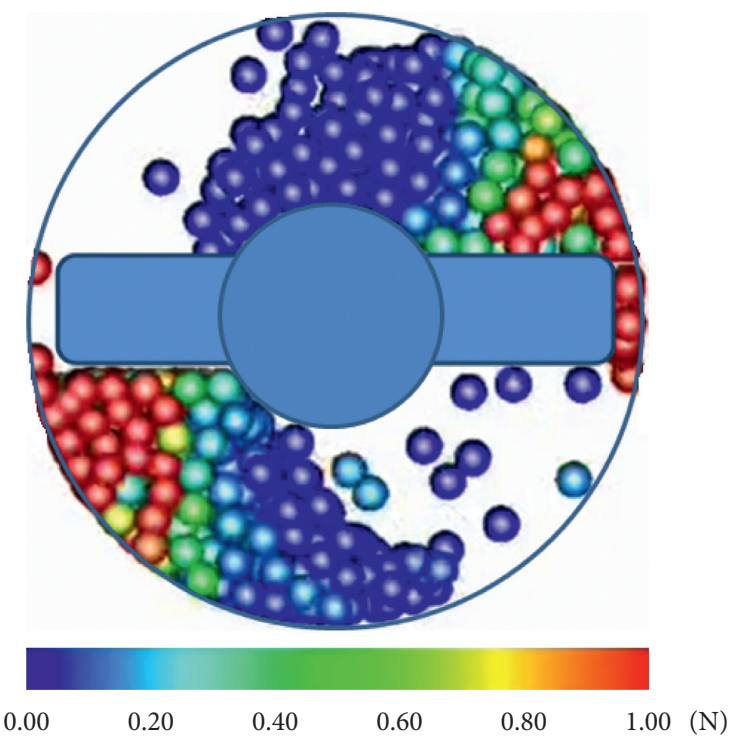

(c)

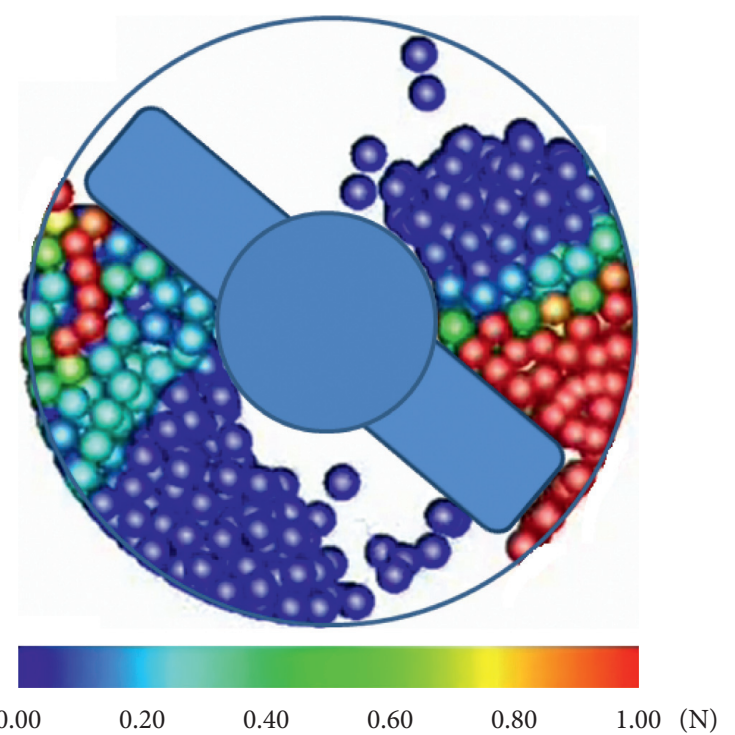

(b)

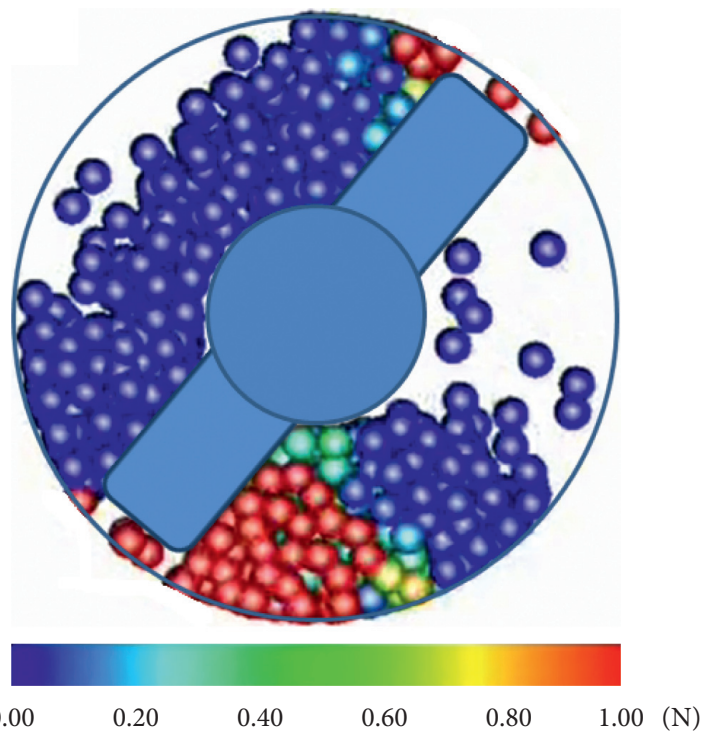

(d)

Figure 9: Compressive force acting on the particle inside the rotary damper with the gap model; the packing fraction is $50 \%$, and the rotational speed is $60 \mathrm{rpm}$. (a) 0 degree, (b) 45 degrees, (c) 90 degrees, and (d) 135 degrees. The direction of rotation is counter-clockwise.

When there are void gaps, particles do not apply strong frictional forces on each other and the walls of the damper [18]. However, when the void gaps are large, the particles are not strongly compressed by neighboring particles, so particles produce weak repulsive forces. The elastic deformation of elastomer particles inside the damper plays a vital role in increasing the damper torque. In short, when there are fewer void gaps inside the damper, particles can produce strong damper torque. As illustrated in Figures 10 and 11, the damper torque increases not only because of the packing fraction but also the rotational speed of the rotor. One of the important factors which cause an increase in damper torque with the increase of rotor speed is the normal and tangential forces acting on the elastomer particles which are strong at high speed of the rotor. The second reason is the increase in the number of collisions between particle-particle and particle-wall. A high-speed rotor pushes the elastomer particles with higher velocity, and particles collide with neighboring particles with high velocity, and loss of energy takes place. In Figure 11, until $10 \mathrm{rpm}$ of the shaft, the damper torque of rotary damper with the gap between rotor and cylinder is higher than the rotary damper with no gap between rotor and cylinder. After $10 \mathrm{rpm}$ of the shaft, the rotary elastomer damper with no-gap model has higher damper torque than the rotary damper with gap model using $3 \mathrm{~mm}$ diameter particles. From 1 to $10 \mathrm{rpm}$, the gap model produced higher damper torque, because at low speed of rotor, particles were crossing the gap region by rolling motion. Due to rolling motion, particles were experiencing compression. At the high rpm of the shaft, the particles 


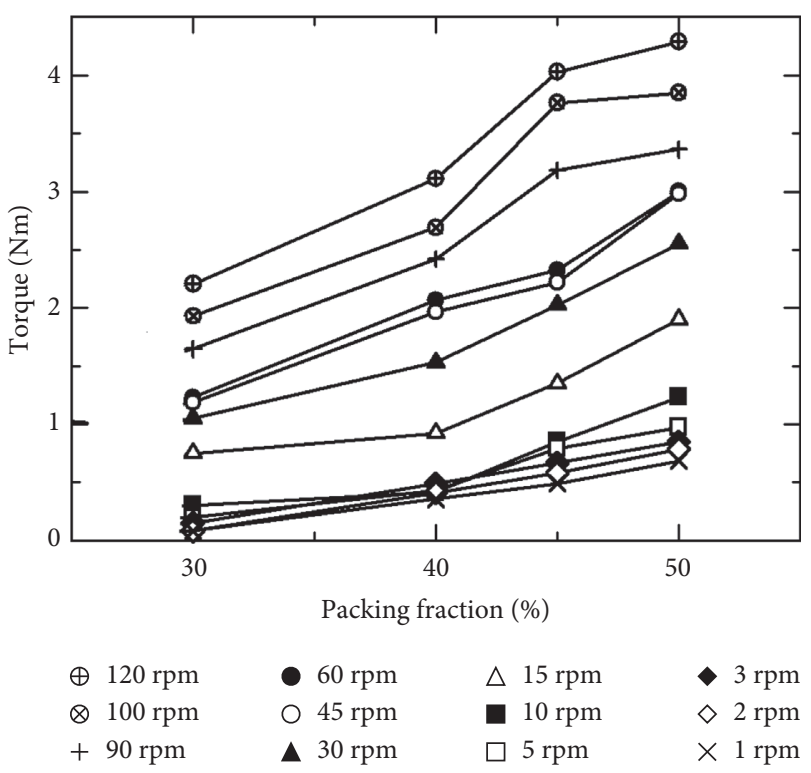

FIGURE 10: The torque versus packing fraction curve of the rotary damper with no gap between the rotor and cylinder using $3 \mathrm{~mm}$ diameter particle made from TSE3466.

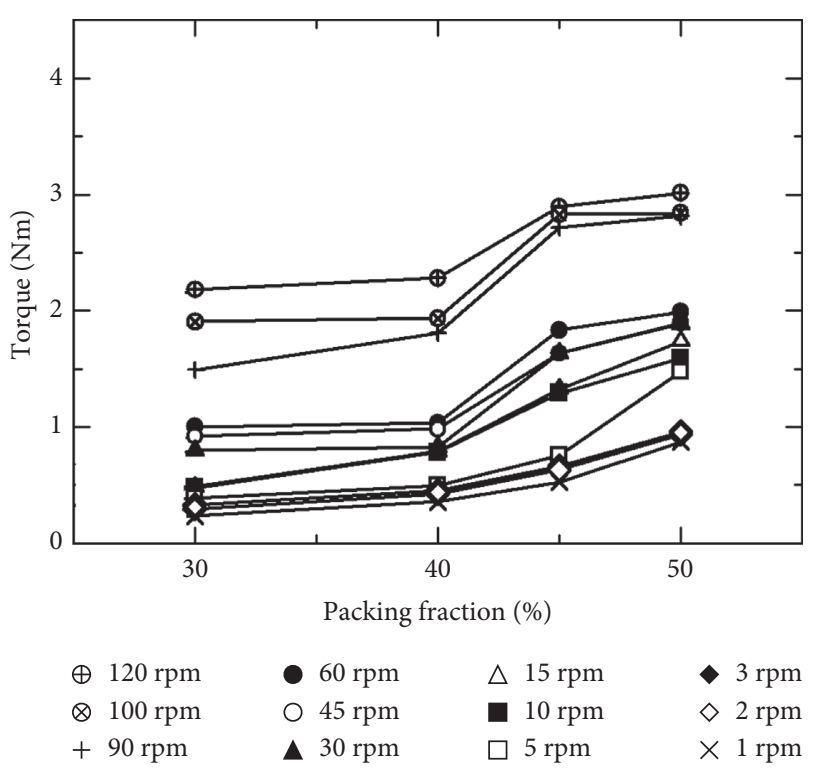

FIGURE 11: The torque versus packing fraction curve of the rotary damper with gap between the rotor and cylinder using $3 \mathrm{~mm}$ diameter particle made from TSE3466.

rolling motion converted to sliding motion when passing through the gap region. In conclusion, due to sliding motion at high rpm, compressive force on particles reduced; that is why after $10 \mathrm{rpm}$, the no-gap model's damper torque is higher than the rotary damper with gap model.

It is worth mentioning that an increase in packing fraction and the rotational speed increases the damper torque, but it has certain limitations. At high rotational speeds such as 100 and $120 \mathrm{rpm}$, the particle breaking rate is higher. Similarly, high rpm of the rotor for a long period can also break the particles.
Previous studies showed that, after a certain packing fraction, a decline in the damping is observed in metallic particle dampers, but in the case of elastomer damper until $50 \%$ packing fraction, the damper torque kept on increasing. We could not observe the limit of packing fraction after which the damper torque may reduce in rotary elastomer particle damper too, because at high packing fractions such as $70 \%$ and above, it is difficult to close the cylinder.

4.3. Effect of Change in Size of Particles on Damper Torque of the Damper. The particles of $3 \mathrm{~mm}, 4 \mathrm{~mm}$, and $5 \mathrm{~mm}$ made from TSE3466 were experimentally tested. This section of the study demonstrates a correlation between the damper torque and the size of elastomer particles. Although the mass ratio is kept constant, the number of particles would vary. In the case of the gap and no-gap models, increase in damper torque was observed with the increase of the size of the elastomer particles. Figure 12 shows the collective results of $3 \mathrm{~mm}, 4 \mathrm{~mm}$, and $5 \mathrm{~mm}$ diameter particles at $60 \mathrm{rpm}$. It is observed that the size of elastomer particles plays a significant role in increasing the damper torque of a rotary elastomer particle damper. A significant increase in damper torque is obtained when $5 \mathrm{~mm}$ diameter elastomer particles are used. The main reason behind the increase in the damper torque with the increase of particle size is the higher deformation capability in large-sized particles. Due to collision and friction between particles and damper body, the velocity of the adjacent particles drops. Particle velocity drops when two particles collide with each other and compares each other. The greater energy dissipation and damping take place when collision and compression are transferred to neighboring particles. Large particles have more contact area and can deform more than small particles under a compressed state. In brief, large size particles tend to produce strong repulsive forces and so result in strong damper torque. It is worth mentioning that when $5 \mathrm{~mm}$ diameter particles are used inside the rotary damper with gap mode, it produces higher damper torque than the rotary damper with the nogap model. When particles of $5 \mathrm{~mm}$ diameters entered into the gap, $5 \mathrm{~mm}$ particles were under intense compressive force due to larger deformation capability. In summary, unlike $3 \mathrm{~mm}$ and $4 \mathrm{~mm}$ diameter particles, $5 \mathrm{~mm}$ particles are in a strongly compressed state even at high rpm, resulting in a strong damper torque generation.

\subsection{Effect of Gap between the Rotor and Cylinder on Damper} Torque of the Damper. In order to investigate the effect of the gap between rotor and cylinder, rotary elastomer particle dampers with gap and no gap between rotor and cylinder are used in this paper. Some of the interesting and unique results are obtained from the study. As illustrated in Figure 13, damper torque of rotary damper with gap model using $3 \mathrm{~mm}$ diameter particles is higher until the $10 \mathrm{rpm}$ of the shaft. After $10 \mathrm{rpm}$ of the shaft, the decrease in the damper torque of the rotary damper with the gap model compared to the no-gap model is due to the sliding of particles through the gap. At the low rotational speed of the shaft, particles slowly cross from the gap between the rotor and the body of the 


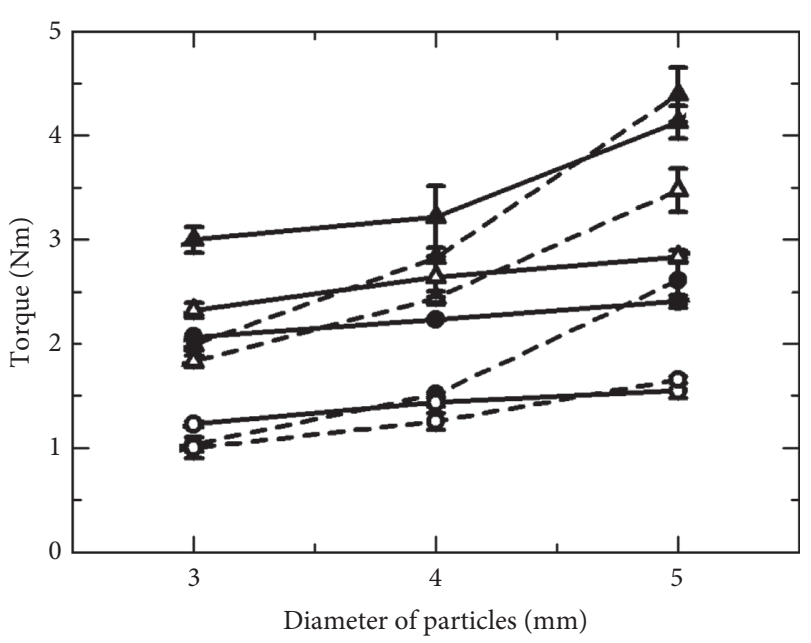

- $50 \%$ Packing

$\triangle 45 \%$ Packing

$40 \%$ Packing

○ $30 \%$ Packing

- No-gap model
--- Gap model

Figure 12: The torque versus diameter of the particle curve of the rotary damper with gap and no-gap models at $60 \mathrm{rpm}$.

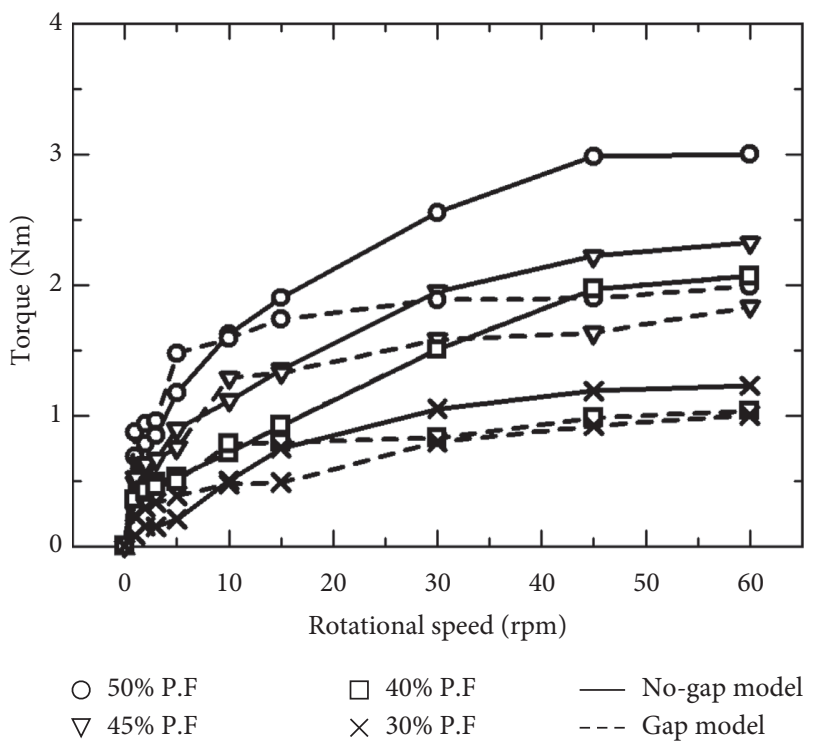

FIGURE 13: The torque versus rotational speed curve of the rotary damper with gap and no gap using $3 \mathrm{~mm}$ diameter elastomer particles.

damper. Due to the rotational motion of particles, strong frictional forces are generated. This rotation of particles turns into a sliding motion when the rotor strongly pushes particles at $\mathrm{rpm}$ (more than $10 \mathrm{rpm}$ ). In summary, particles do not go through enormous compression at high rpm while crossing through the gap region. At high rpm, they quickly cross the gap region by sliding, which results in weak compressive forces on the particles. However, in the case of the rotary damper with a no-gap model, there is no gap region, but the neighboring particles support particles in front of the rotor. In the gap model, the particles cross from the gap region, so at high $\mathrm{rpm}$, the neighboring particles' support is weak compared to the rotary damper with no gap.

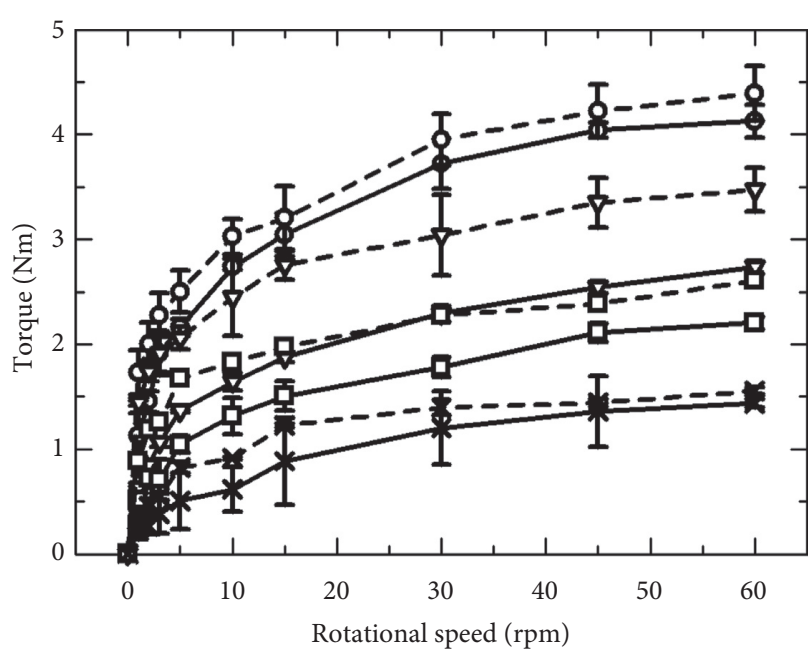

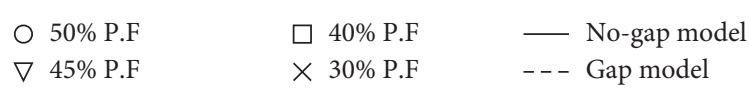

FIGURE 14: The torque versus rotational speed curve of the rotary damper with gap and no-gap models using $5 \mathrm{~mm}$ diameter elastomer particles.

In Figure 14, interesting results are obtained when elastomer particles of $5 \mathrm{~mm}$ diameter are used. Interestingly, the damper torque of the rotary damper with the gap model is larger than the rotary damper with the no-gap model when $5 \mathrm{~mm}$ diameter particles are used. Because of the higher deformation capability of $5 \mathrm{~mm}$ diameter particles compared to $4 \mathrm{~mm}$ and $3 \mathrm{~mm}$ diameter particles, $5 \mathrm{~mm}$ diameter particles have more contact area, and they can deform more under a compressed state. In conclusion, $5 \mathrm{~mm}$ diameter elastomer particles tend to produce strong repulsive forces, resulting in strong damper torque. In summary, unlike $3 \mathrm{~mm}$ and $4 \mathrm{~mm}$ diameter particles, $5 \mathrm{~mm}$ particles are in the strongly compressed state even at high rpm, which results in the generation of a strong damper torque.

4.5. Findings and Significance in Comparison to Existing Approaches. In this subsection, we discuss the unique and significant findings of this study by comparing it with the state-of-the-art approaches.

(1) Usually, particle dampers consist of metallic particles, but metallic particles produce enormous noise due to collision between particle-particle and particle-wall [13]. In this study, we used elastomer particles instead of metallic particles. Elastomer particles do not produce noise due to collision between particle-particle and particle-wall because of their soft nature.

(2) Research on metallic particles showed that after a specific diameter of particles, further increase in the size of particles reduced the damping performance [12]. However, we used elastomer particles of $3 \mathrm{~mm}$, $4 \mathrm{~mm}$, and $5 \mathrm{~mm}$ diameter; no reduction in damper torque is observed with an increase in the size of elastomer particles. 
(3) Generally, in metallic particles, after a specific limit of packing fraction, further increase in the packing fraction reduced the damping capabilities of the damper $[9,10,12,16]$. However, we used elastomer particles, and no reduction in damper torque is noticed with the increase in the packing fraction.

(4) This study proved that particle deformation plays a significant role in producing higher damping in elastomer particle dampers. The elastomer particles in front of the rotor play a vital role in producing strong damper torque because they have strong compressive forces.

(5) Gap and no gap between the rotor and the cylinder with respect to different sizes of elastomer particles have not been studied in previous studies. In this research, we proved that to obtain higher damping, it is recommended to use a no-gap model when elastomer particles with $3 \mathrm{~mm}$ diameter are used inside the damper. However, when the particles size is $5 \mathrm{~mm}$, the damper with the gap between rotor and cylinder can produce higher damping.

\section{Conclusion}

We investigated the effect of change in packing fraction, rotor speed, size of elastomer particles, and the gap between the rotor and cylinder on the damper torque. Prototypes of the rotary elastomer particle damper with gap and no gap between rotor and cylinder were introduced to observe the damper torque performance of rotary elastomer damper. Spherical elastomer particles made from TSE3466 are tested in the rotary damper with $3 \mathrm{~mm}, 4 \mathrm{~mm}$, and $5 \mathrm{~mm}$ diameters. An increase in damper torque is observed by increasing the packing fraction. Particles made of TSE3466 were tested to observe the effect of the particles' size on damper performance. It is proved that $5 \mathrm{~mm}$ diameter particles showed higher damper torque than $4 \mathrm{~mm}$ and $3 \mathrm{~mm}$ diameter particles. Interestingly, the damper torque of the rotary damper with the gap model was higher than the no-gap model until $10 \mathrm{rpm}$ of the shaft when $3 \mathrm{~mm}$ diameter elastomer particles were used. The damper torque of the rotary damper with the gap between rotor and cylinder is larger than the rotary damper with a no-gap model throughout any rpm when $5 \mathrm{~mm}$ diameter elastomer particles are used.

\section{Data Availability}

The data supporting the findings of this study are available within the article. Further, any specific data will be provided by the corresponding author upon reasonable request.

\section{Consent}

This article does not contain any studies with human participants.

\section{Conflicts of Interest}

The authors declare that they have no conflicts of interest.

\section{References}

[1] R. Kawamoto, Y. Ido, and A. Toyouchi, "Damping properties of a damper using an elastomer particle assemblage containing fine particles," Advanced Experimental Mechanics, vol. 1-10, pp. 99-104, 2016.

[2] X. Li, Y. Yang, and W. Shi, "Study on the damping effect of particle dampers considering different surface properties," Shock and Vibration, vol. 2019, Article ID 8293654, 16 pages, 2019.

[3] Z. Lu, X. Lu, H. Jiang, and S. F. Masri, "Discrete element method simulation and experimental validation of particle damper system," Engineering Computations, vol. 31, no. 4, pp. 810-823, 2014.

[4] Z. Lu, Z. Wang, S. F. Masri, and X. Lu, "Particle impact dampers: past, present, and future," Structural Control and Health Monitoring, vol. 25, no. 1, 2018.

[5] H. V. Panossian, "Structural damping enhancement via nonobstructive particle damping technique," Journal of Vibration and Acoustics, vol. 114, no. 1, pp. 101-105, 1992.

[6] K. S. Marhadi and V. K. Kinra, "Particle impact damping: effect of mass ratio, material, and shape," Journal of Sound and Vibration, vol. 283, no. 1-2, pp. 433-448, 2005.

[7] M. Sánchez, C. M. Carlevaro, and L. A. Pugnaloni, "Effect of particle shape and fragmentation on the response of particle dampers," Journal of Vibration and Control, vol. 20, no. 12, pp. 1846-1854, 2013.

[8] W. Liu, G. R. Tomlinson, and J. A. Rongong, "The dynamic characterisation of disk geometry particle dampers," Journal of Sound and Vibration, vol. 280, no. 3-5, pp. 849-861, 2005.

[9] A. Papalou and S. F. Masri, "Response of impact dampers with granular materials under random excitation," Earthquake Engineering \& Structural Dynamics, vol. 25, no. 3, pp. 253-267, 1996.

[10] R. D. Friend and V. K. Kinra, "Particle impact damping," Journal of Sound and Vibration, vol. 233, no. 1, pp. 93-118, 2000.

[11] F. Duvigneau, S. Koch, E. Woschke, and U. Gabbert, "An effective vibration reduction concept for automotive applications based on granular-filled cavities," Journal of Vibration and Control, vol. 24, no. 1, pp. 73-82, 2018.

[12] P. S. Kachare and K. Bimleshkumar, "Effect of particle size and packing ratio of PID on vibration amplitude of beam," Journal of Mechanical Engineering and Sciences, vol. 4, pp. 504-517, 2013.

[13] H. Ye, Y. Wang, B. Liu, and X. Jiang, "Experimental study on the damping effect of multi-unit particle dampers applied to bracket structure," Applied Sciences, vol. 9, no. 14, 2019.

[14] Y. Morishita, Y. Ido, K. Maekawa, and A. Toyouchi, "Basic damping property of a double-rod type damper utilizing an elastomer particle assemblage," Advanced Experimental Mechanics, vol. 1-21, pp. 93-98, 2016.

[15] K. K. Kishan, Y. Ido, Y. Iwamoto, and A. Toyouchi, "Experimental investigation of angle dependent torque properties of a particle rotary damper using a magnetic elastomer particle assemblage," International Symposium on Applied Electromagnetics and Mechanics, vol. 64, pp. 737-743, 2020.

[16] Z. Lu and M. W. Yan, "A survey of particle damping technology," Zhendong yu Chongji/Journal of Vibration and Shock, vol. 32-7, pp. 1-7, 2013.

[17] B. Darabi and J. A. Rongong, "Polymeric particle dampers under steady-state vertical vibrations," Journal of Sound and Vibration, vol. 331, no. 14, pp. 3304-3316, 2012. 
[18] A. Toyouchi, M. Hanai, Y. Ido, and Y. Iwamoto, "Damper force characteristics of a separated dual-chamber single-rodtype damper using an elastomer-particle assemblage," Journal of Sound and Vibration, vol. 488, Article ID 115625, 2020.

[19] The Japan Society of Mechanical Engineers, JSME Mechanical Engineers' HandbookMaruzen Publishing Co.,Ltd, Tokyo, Japan, 6 edition, pp. 3-34, Tokyo, Japan, 1977, (in Japanese).

[20] K. Alireza and Z. Mansourpour, "Influence of non-spherical shape approximation on DEM simulation accuracy by multisphere method," Powder Technology, vol. 332, pp. 265-278, 2018. 